\title{
Injection therapy for urologic chronic pelvic pain: Lessons learned
}

\author{
J. Curtis Nickel, MD, FRCSC
}

Department of Urology, Queen's University, Kingston, ON, Canada

Cite as: Can Urol Assoc J 2018;12(6Suppl3):S186-8. http://dx.doi.org/10.5489/cuaj.5333

\section{The principle}

Pain is transmitted by afferent nerves from skin, organs, muscle, and other related structures in the lower abdomen and pelvis, while muscle spasm can be maintained by efferent nerves. These nerves are modulated centrally in the nervous system and can become upregulated with subsequent sensitization of the end organ. By injecting the nerves with local anesthetic, we effectively block this process. If we are accurate, the pain resolves, even if temporarily. This fact in itself allows for much better diagnosis of the cause of the patient's pain. However, a phenomenon that can occur by temporarily breaking this pain cycle is that when the pain returns, it may not be as severe as it was before the injection. With repeated injections, we may be able to downregulate or desensitize the chronic pain cycle. While this is a very gross simplification of what we are trying to accomplish, it is the explanation that I provide to all my patients. And they understand the rationale and are willing to give it a try. For practical purposes, my first injection therapy will be a test, at trial to see if the pain resolves. For this first test, I will use half lidocaine $2 \%$ and half bupivacaine $(0.25 \%)$. If that is successful in ameliorating the pain to the patient's satisfaction, I will offer repeat injections (weekly if possible, but sometimes biweekly) with $0.25 \%$ bupivacaine. Table 1 outlines my maximum doses per injection session. I only use a steroid (cortisone or triamcionolone) if I believe that I am dealing with an inflammatory condition and/or nerve entrapment syndrome. Once we start to see success, I space out the injections to monthly and then only as required by the patient.

\section{Pelvic pain syndrome}

The most important nerve system involved in pelvic pain is the pudendal nerve and its branches, which supply the pelvic musculature and perineum (Fig. 1). Neuropathy with central upregulation can lead to areas of point tenderness or widespread (but typically unilateral) pain and painful muscle spasm in the nerve distribution. I used to inject the pelvic floor using a transvaginal (in women) or transrectal (in men) approach with a metal finger protector (scrounged from days when we used to perform freehand transrectal biopsies), but recently have had much more success and found it much simpler to use a transperineal approach, even for trigger point injections.

I start off by palpating the ischial tuberosity and freeze a point between the tuberosity, anus, and posterior vulva with $1 \%$ lidocaine in a tuberculin or diabetic syringe/needle. I then place my index finger into the vagina and palpate any superficial (bulbospongiosus, ischiocavernosus) or deeper (pubo/iliococcygeus) muscle spasm/pain, as well as any trigger pain points. I then advance a 22 gauge, 3.5 inch spinal needle through my preselected perineal site, aimed towards the ipsilateral shoulder and ischial spine (Fig. 2). To mitigate injury to my index finger, once I have determined the ideal needle path, I pull my finger away from the vaginal wall on that side as I advance the needle tip. I slowly insert the needle to its hilt, which should land me at or near the pudendal nerve after it has exited Alcocks canal as it branches through the pelvis. I remove the internal needle stylet, attach a 10 cc syringe, and then slowly inject 0.5-1.0 cc of the chosen anesthetic solution at that site (after aspirating for blood). I then slowly retract the needle, injecting as I gently pull it out, sometimes repositioning it so that I can obtain a "fan" injection pattern. I sometimes reposition the needle laterally to take care of the more superficial perineal branch as well. I inject a total of $10 \mathrm{cc}$ on each affected side (many times I only have to perform a unilateral injection). If done gently while talking to the patient and explaining what you are doing, it can be very well-tolerated and patients who have had significant improvement, even if short-lived, will want to come back for repeated injections.

The technique is similar in men, however, the index finger in the rectum is used to direct the needle path. When trying to decide if the patient has a prostate pain syndrome (many times the men will describe a painful golf or tennis ball sensation in the perineum) as opposed to a pelvic floor or pudendal nerve problem, I will inject the peri-prostatic area (being sure to get to the base of the prostate) using a blind hand-directed approach. It seems to work well for me, however, I am sure that a urologist who has a level of comfort with transrectal ultrasound will achieve a more accurate placement of anesthetic as performed for transrectal biopsies 
Table 1. Maximum dose of local anesthetics

\begin{tabular}{l} 
Note: The maximum dose of local anesthetics must never be \\
exceeded at any treatment session. My usual dose of $0.25 \%$ \\
bupivacaine per session rarely exceeds $20 \mathrm{cc}$ \\
\hline Maximum dose of lidocaine is $300 \mathrm{mg}$ \\
$-30 \mathrm{cc}$ of $1 \%$ lidocaine \\
$-15 \mathrm{cc}$ of $2 \%$ lidocaine \\
Maximum dose of bupivacaine (marcaine) is $150 \mathrm{mg}$ \\
$-25 \mathrm{ml}$ of $0.5 \%$ bupivacaine \\
$-50 \mathrm{cc}$ of $0.25 \%$ bupivacaine
\end{tabular}

and may have an even better outcome. Again, since I am using my gloved index finger to determine the needle path, when advancing the needle, I pull my finger away from either the prostate gland (prostatic block) or that side of the rectum (pelvic nerve block) to prevent my finger being pricked by the needle. The procedure is very well-tolerated by men as long as the procedure is done slowly while explaining each step to the patient as the procedure is being done.

\section{Scrotal pain syndrome}

It is important to have some idea of the nerves that are involved in scrotal, epididymal, and testicular pain. Fig. 3 shows the various nerves involved, which include the spermatic nerves initiating from $\mathrm{T} 10 \mathrm{~L} 1$, which course through a complicated path via the superior mesenteric, hypogastric, and then pelvic nerve plexus before finding its way in the spermatic cord to the scrotal contents. But as depicted in Fig. 3, the ilioinguinal (and iliohypogastric), both branches of the genitofemoral nerve, as well as the perineal branch of the pudendal nerve, all are involved in scrotal sensation.

The afferent nerve fibres for the internal contents of the scrotum run in the spermatic cord. I have found that the easiest approach to anesthesia of the testicle and epididymis is by grasping the spermatic cord between two fingers after it exits from the inguinal canal (Fig. 4) and injecting directly into cord with 6-8 cc of local anesthetic. The genital branch of the genitofemoral nerve runs just outside the cord and can be anesthetized by injecting just lateral to the cord as it emerges from the external inguinal ring, but this in not required for management of orchiodynia. The pudendal and/ or ilioinguinal nerves do not have to be injected for orchiodynia evaluation or therapeutic desensitization. These nerves can be injected if you want to establish anesthesia of the overlying scrotal skin.

\section{Inguinal and abdominal wall pain syndromes}

Inguinal and abdominal wall pain (suprapubic, rectus sheath, etc.) can be managed with direct injection into painful trigger points. These trigger points can be determined by physical examination, but most often are accurately pointed out by the patient. Once the trigger point site is determined, the

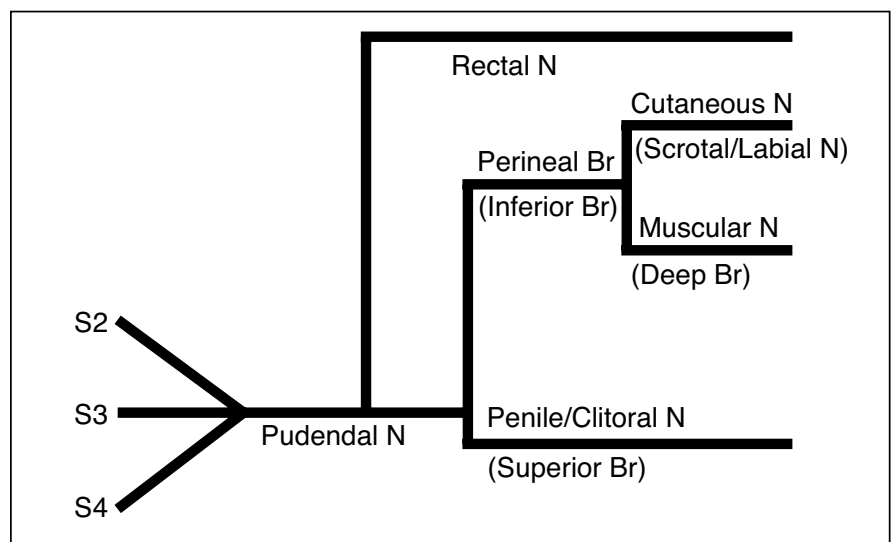

Fig. 1. Pelvic nerve distribution.

needle injection must be deep enough to deposit anesthetic into the external, internal, and even traversus abdominus muscle using a "fan" type of injection pattern.

The spermatic branches of the ilioinguinal nerve can be blocked at the level of the spermatic cord in the inguinal canal by doing a high nerve block of the spermatic cord. It is sometimes difficult to find the exact location for a successful block and may not be helpful for most urologic chronic pain conditions (the low spermatic cord block, as described in the previous section, is much easier to accomplish). A high block of the ilioinguinal and hypogastric nerves can be accomplished by injection at a site $2 \mathrm{~cm}$ medial and 2

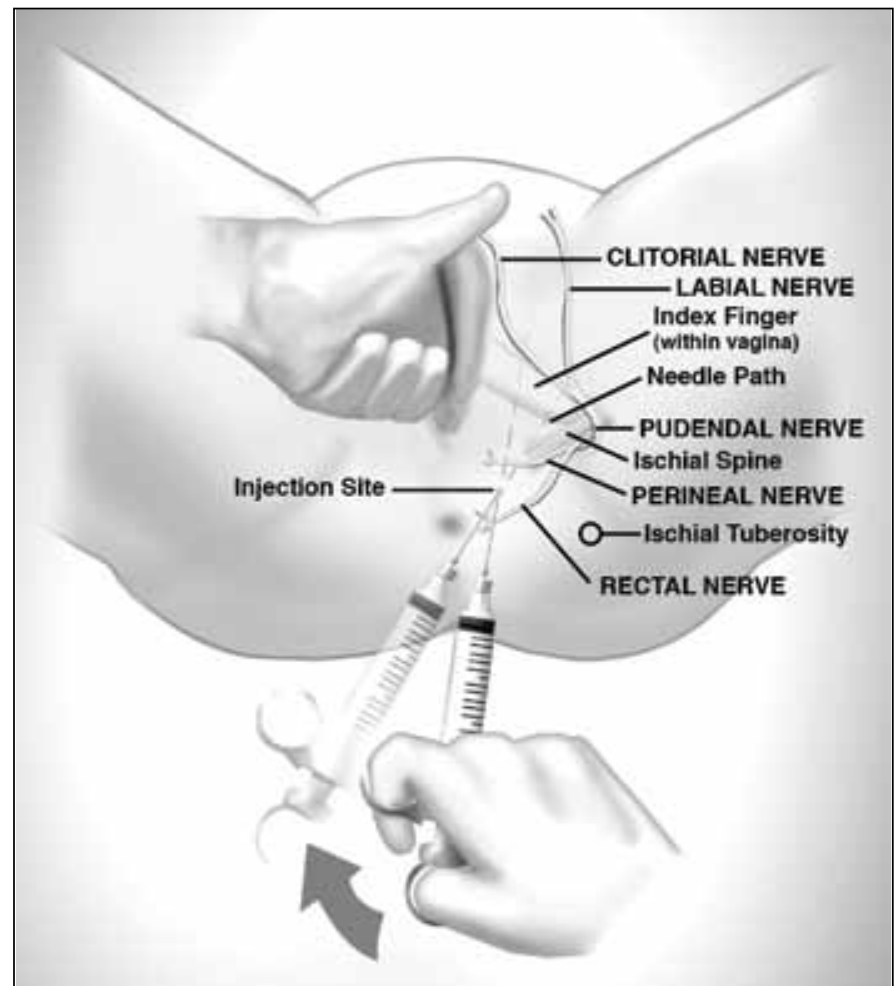

Fig. 2. Technique of pelvic floor injection. 


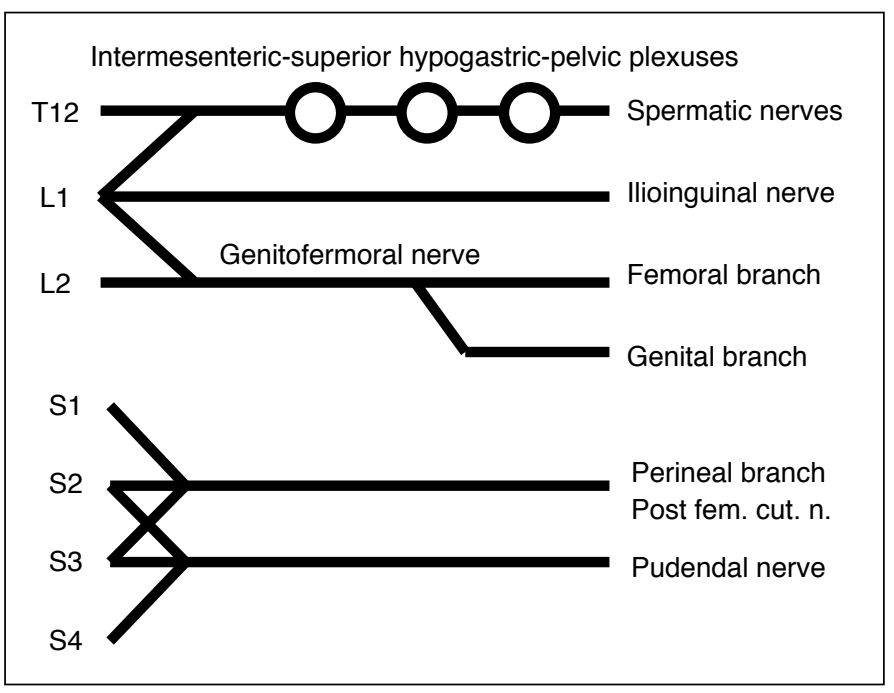

Fig. 3. Scrotum and scrotal content nerve distribution.

$\mathrm{cm}$ inferior from the anterior superior iliac spine (although I know of others who inject a much more superior abdominal wall point described as $2 \mathrm{~cm}$ medial and $2 \mathrm{~cm}$ caudad), making sure that the anesthetic solution is injected between the internal oblique and external oblique muscles, as well as between the internal oblique and transversus abdominua. To accomplish this, I have learned to appreciate the increased resistance as the needle encounters the fascia covering the external oblique muscle and a loss of resistance ("pop" sensation) as it passes into the internal oblique muscle. Passing through the next resistance point, the needle finally enters the transversus abdominus muscle and $10 \mathrm{cc}$ of anesthetic is injected in a fan-like manner so that a reasonably wide area between the internal oblique and transversus abdominus muscle, as well as between the external and internal oblique, is infiltrated.

For pain related to the rectus abdominis muscle or rectua sheath insertions, I use a blind technique to inject the local anesthetic on the posterior wall of the rectus sheath (the terminal branches of the intercostal nerves penetrate the posterior wall of the rectus abdominis muscle). Again, the resistance of the sheath is my landmark and one can feel a definitive "pop" when it passes through. Of course, care must be taken, particularly with rectus sheath injections, to miss the peritoneal cavity. Fig. 4 illustrates these various abdominal wall injection sites.

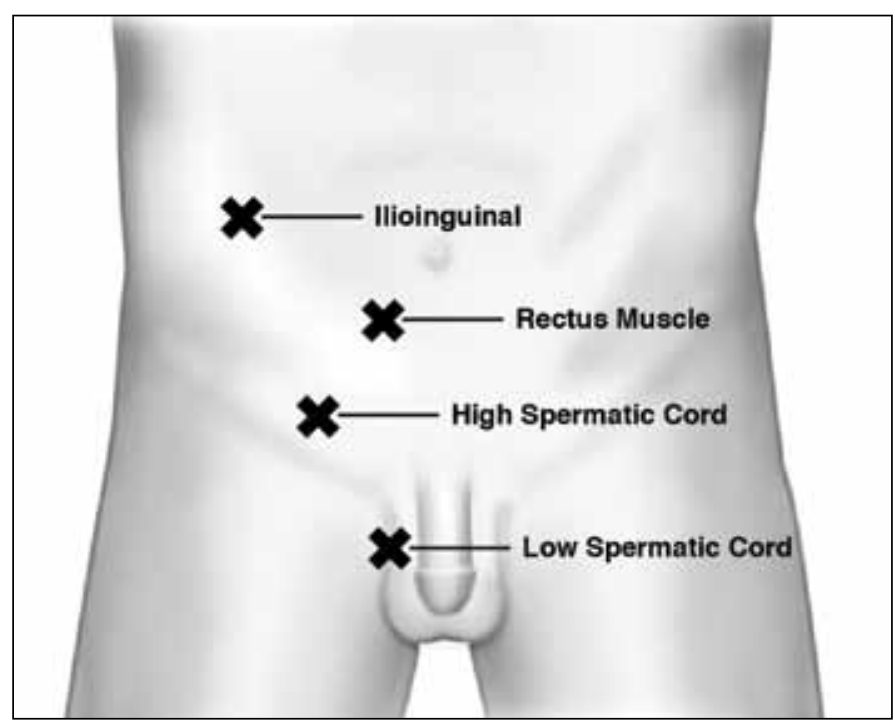

Fig. 4. Injection site for scrotal, inguinal, and abdominal wall injections.

\section{Final lesson learned}

I learn something new from every one of my patients who have embarked on an injection therapy course of treatment. From some I learn the little nuances of careful advancement of the needle, from others I have learned that not everyone's anatomy is the same, while I have learned that all my patients appreciate the fact that I am aggressively attempting to manage the pain syndrome that has so impacted their life. I find that this is true, even if it was not a success in their particular case. At least I tried. I have found it to be satisfying to see patients slowly improve with serial anesthetic injections. I hope that some of the painful lessons that I have learned will provide some guidance to urologists who want to have that same sense of satisfaction in helping our most disadvantaged patients in urology practice.

Competing interests: Dr. Nickel has been a consultant for Astellas, Auxillium, Eli Lilly, Farr Labs, Ferring, GSK, Pfizer, Redleaf Pharma, Taris Biomedical, Tribute, and Trillium Therapeutics; a lecturer for Astellas and Eli Lilly; and has participated in clinical trials supported by Eli Lilly, GSK, J\&J, Pfizer, and Taris Biomedical.

This paper has been peer reviewed.

Correspondence: Dr. J. Curtis Nickel, Department of Urology, Queen's University, Kingston, ON, Canada; jin@queensu.ca 